\title{
Clinically adaptable polymer enables simultaneous spatial analysis of colonic tissues and biofilms
}

\author{
Mary C. Macedonia ${ }^{1,2}$, Julia L. Drewes $\mathbb{D}^{3}$, Nicholas O. Markham $\mathbb{D}^{1,2}$, Alan J. Simmons ${ }^{2,4}$, Joseph T. Roland ${ }^{2,5}$, Paige N. Vega $\mathbb{D}^{2,4}$, \\ Cherie' R. Scurrah $^{2,4}$, Robert J. Coffey ${ }^{1,2,6}$, Martha J. Shrubsole $\mathbb{D}^{6,7}$, Cynthia L. Sears $\mathbb{1 D}^{3}$ and Ken S. Lau (iD) ${ }^{2,4,6 凶}$
}

Microbial influences on host cells depend upon the identities of the microbes, their spatial localization, and the responses they invoke on specific host cell populations. Multimodal analyses of both microbes and host cells in a spatially resolved fashion would enable studies into these complex interactions in native tissue environments, potentially in clinical specimens. While techniques to preserve each of the microbial and host cell compartments have been used to examine tissues and microbes separately, we endeavored to develop approaches to simultaneously analyze both compartments. Herein, we established an original method for mucus preservation using Poloxamer 407 (also known as Pluronic F-127), a thermoreversible polymer with mucus-adhesive characteristics. We demonstrate that this approach can preserve spatially-defined compartments of the mucus bi-layer in the colon and the bacterial communities within, compared with their marked absence when tissues were processed with traditional formalinfixed paraffin-embedded (FFPE) pipelines. Additionally, antigens for antibody staining of host cells were preserved and signal intensity for $16 \mathrm{~S}$ rRNA fluorescence in situ hybridization (FISH) was enhanced in poloxamer-fixed samples. This in turn enabled us to integrate multimodal analysis using a modified multiplex immunofluorescence (MxIF) protocol. Importantly, we have formulated Poloxamer 407 to polymerize and cross-link at room temperature for use in clinical workflows. These results suggest that the fixative formulation of Poloxamer 407 can be integrated into biospecimen collection pipelines for simultaneous analysis of microbes and host cells.

npj Biofilms and Microbiomes (2020)6:33; https://doi.org/10.1038/s41522-020-00143-x

\section{INTRODUCTION}

Investigating host-microbe interactions may reveal the mechanisms underlying how changes in the gastrointestinal microbiome are related to colorectal cancer (CRC). Recently, increased abundance of Fusobacterium nucleatum, Bacteroides fragilis, and Escherichia coli have been associated with human $\mathrm{CRC}^{1-3}$. These species are hypothesized to promote tumorigenesis and/or progression of CRC through upregulation of oncogenes, modification of intestinal mucus, or damage to host $\mathrm{DNA}^{2,4,5}$. In addition to the mere presence or absence of putative carcinogenic bacteria in the gut, the spatial localization and physical interaction with host epithelial cells may be critically important.

Bacterial biofilms are ubiquitous in nature and can also be observed routinely in the gastrointestinal tract of healthy individuals specifically in the outer layer of the MUC2dominated mucus bi-layer that lines the luminal colonic surface ${ }^{6-11}$. In contrast, the inner, striated mucus layer of the colon is largely sterile in healthy hosts ${ }^{6}$. Perturbations of this tightly regulated spatial organization have been linked to both inflammatory bowel diseases and CRC ${ }^{12-15}$. Mucosa-associated bacterial biofilms are defined as microbial aggregates embedded within a polymeric matrix of both host and bacterial origin that directly contacts epithelial cells ${ }^{14,16}$. Mucosa-associated biofilms have been identified in $\sim 50 \%$ of sporadic CRC patients, $100 \%$ of familial adenomatous polyposis (FAP) patients, and $13 \%$ of healthy individuals. They are particularly enriched in CRCs from the ascending colon ${ }^{3}$. Additionally, bacterial isolates from CRC or FAP biofilms have been shown to promote colonic tumor progression in genetically susceptible mice ${ }^{13,15}$.

While many studies have characterized the microbiome through sequencing and/or mass spectrometry, host-microbe spatial relationships can only be studied with sufficient resolution through microscopy-based techniques, such as fluorescence in situ hybridization (FISH) or electron microscopy ${ }^{11,12}$. Moreover, while sophisticated multimodal analyses are being conducted on the microbiome ${ }^{17}$, concurrent analyses of host cells have lagged. Studies on the host response to microbiome alterations to date have typically relied on macroscopic outputs, such as tumor formation, or molecular assays performed on separate, spatially disconnected specimens ${ }^{2,15}$. We propose that concurrent, multimodal analysis of both host and microbial cells within the same tissue specimen would significantly advance knowledge into host-microbe interactions in CRC, as well as other forms of pathogenesis.

Recently developed imaging techniques enable multiplexed in situ analysis of 50 or more analytes on the same tissue section ${ }^{18-27}$. However, one challenge has been identifying a tissue processing method that enables simultaneous spatial localization and quantifiable detection of both host components, such as proteins, and bacterial components via immunofluorescence (IF) and FISH, respectively. The colonic mucus layer acts as both a resident niche and a barrier for the luminal microbiome, playing well-documented roles in colonic pathologies such as ulcerative colitis $^{10,14,28,29}$. The hydrophilic colonic mucus is easily disrupted

\footnotetext{
${ }^{1}$ Division of Gastroenterology, Hepatology and Nutrition, Department of Medicine, Vanderbilt University Medical Center, Nashville, TN, USA. ${ }^{2}$ Epithelial Biology Center, Vanderbilt University Medical Center, Nashville, TN, USA. ${ }^{3}$ Division of Infectious Diseases, Department of Medicine, Johns Hopkins University School of Medicine, Baltimore, MD, USA. ${ }^{4}$ Department of Cell and Developmental Biology, Vanderbilt University School of Medicine, Nashville, TN, USA. ${ }^{5}$ Department of Surgery, Vanderbilt University Medical Center, Nashville, TN, USA. ${ }^{6}$ Vanderbilt Ingram Cancer Center, Nashville, TN, USA. ${ }^{7}$ Division of Epidemiology, Vanderbilt Epidemiology Center, Department of Medicine, Vanderbilt University Medical Center, Nashville, TN, USA. ${ }^{凶}$ email: ken.s.lau@vanderbilt.edu
} 
by hydration and dehydration of formalin-fixed tissue with standard histological preparations ${ }^{7,30}$. Carnoy's solution is a rapidly dehydrating fixative that preserves mucus ${ }^{9,14}$, but it reduces the sensitivity of IF and $\mathrm{FISH}^{7,31}$. Our goal for this work is to devise a tissue processing strategy that preserves mucus architecture and is amenable to coincident antibody and nucleic acid detection. Herein, we describe how Poloxamer 407 fixation permits the simultaneous characterization of host cell protein expression and identification of microbial communities within the colonic mucus. Furthermore, we deployed this approach into a pilot clinical pipeline to enable multiplex in situ profiling of human tissues and associated microbes.

\section{RESULTS}

Carnoy's and formalin fixation are incompatible with simultaneous mucus preservation and host cell assays

The alcohol-based Carnoy's solution (Carnoy's fixative/Methacarn) is a rapid dehydration fixative that preserves the hydrophilic mucus layer for colonic microbial FISH analysis. Cross-linking fixative, such as neutral buffered formalin (NBF), is used for standard formalin-fixed paraffin-embedded (FFPE) tissue processing and has optimal antigen preservation for IF or immunohistochemistry ${ }^{32,33}$. For this study, we aimed to identify the best tissue fixation method for simultaneous microbial FISH and host cell IF. We initially used mouse colon tissue as a model because of its abundance and availability. Mice do not naturally form mucosaassociated biofilms, but their mucus layers are arranged in bi-layer structures with different microbial compositions that can easily be evaluated.

Initially, we compared Carnoy's fixation and NBF fixation, followed by standard histological processing. Standard IF stains such as proliferating cell nuclear antigen (PCNA) and the epithelial marker pan-cadherin (PCAD) had lower fluorescent signals in Carnoy's- and Methacarn-fixed mouse colons compared to NBF fixation (Fig. 1a, b and Supplementary Fig. 1). In contrast, NBF fixation results in loss of the mucopolysaccharide-containing mucus layer (Fig. 1C), because it cannot withstand hydration and shear forces during processing. NBF contains mostly water and solubilizes the hydrophilic mucus layer during the fixation process $^{11,34,35}$. Consistent with known knowledge, these conventional fixatives are inadequate for in situ analysis of mucusresiding bacteria and host cells within the same tissue section.

Scaffolding agents have been used to maintain the mucus layer during formaldehyde-based fixation ${ }^{36}$. Thus, we embedded freshly harvested mouse colon with low melting point agarose gel to scaffold the mucus while fixing the tissue with $4 \%$ paraformaldehyde at room temperature (Fig. 1d). The mucus layer was retained, consistent with previous studies, but its morphology was distorted by hydration. This modest improvement prompted us to identify substrates for scaffolding the mucus layer, including those that can chemically adhere and cross-link mucus.

Poloxamer 407 formulated as a fixative solution polymerizes at room temperature

Poloxamer 407 is a bio-adhesive polymer with an A-B-A block configuration, with a hydrophobic polypropylene oxide " $B$ " block flanked by hydrophilic polyethylene oxide "A" blocks (Fig. 2a, b). Poloxamer 407 exhibits temperature-dependent and reversible polymerization, essentially transforming from a viscous liquid at $4{ }^{\circ} \mathrm{C}$ to a solid gel at higher temperatures capable of scaffolding biological surfaces. As temperature increases, hydrophobic B blocks align at the core while hydrophilic A blocks are oriented outward in a radial pattern to form micelles that can been used for nanoparticle drug delivery ${ }^{37-40}$. At the ceiling temperature, Poloxamer 407 forms a matrix of micelles held together by hydrogen bonds (Fig. 2c) ${ }^{37,41}$, with the outwardly arranged hydrogen molecules capable of forming non-covalent bonds with the highly glycosylated mucus matrix (Fig. 2d) ${ }^{39}$. Because of this

\section{Antibody Comparison}

\section{Carnoy's Solution}
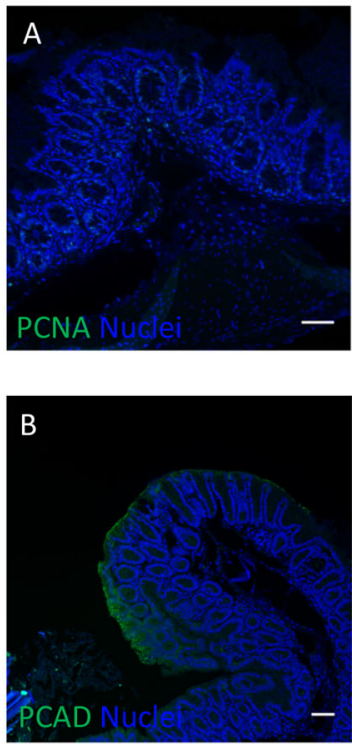

NBF
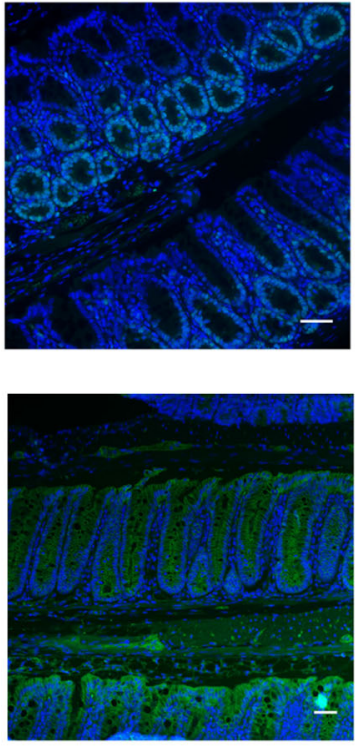

Mucus Analysis

Carnoy's Solution
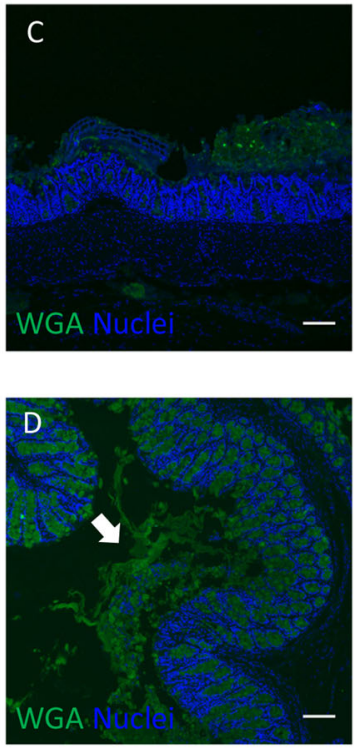
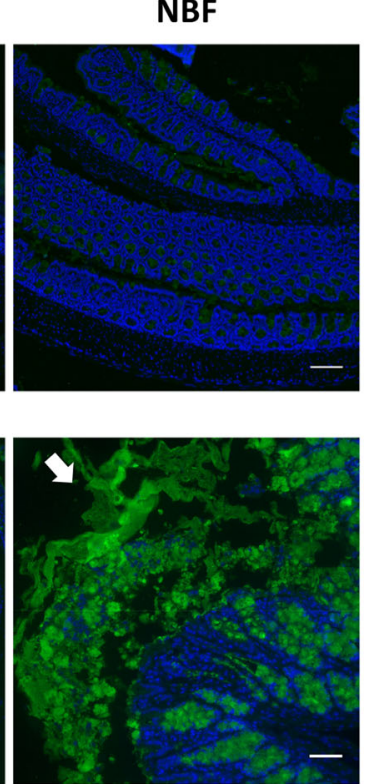

Fig. 1 Carnoy's and formalin fixation preclude multimodal analysis of colonic tissues with intact mucus. Comparison of protein staining using IF between mouse colonic tissues fixed with Carnoy's solution (left) or NBF (right) for $\mathbf{a}$ PCNA and $\mathbf{b}$ pan-cadherin (PCAD). Scale bars = $50 \mu \mathrm{m}$. c Mucus layer preservation as assessed by lectin (WGA) IF of mouse colonic tissues processed with Carnoy's solution (left) or NBF (right). Scale bars $=100 \mu \mathrm{m}$. d Mucus layer preservation using low melting point agarose as a scaffold. White arrows point to distorted portions of the mucus layer. Left-low magnification view. Scale bar $=100 \mu \mathrm{m}$. Right—high magnification view. Scale bar $=50 \mu \mathrm{m}$. 


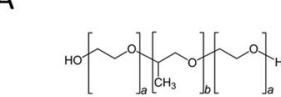

B

C

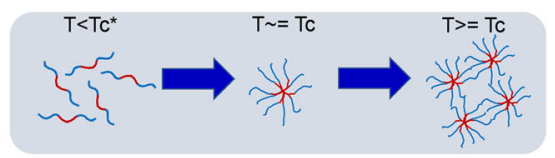

D

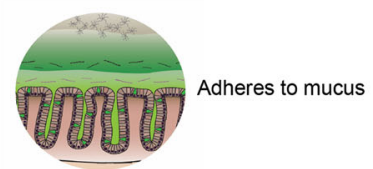

$\mathrm{E}$

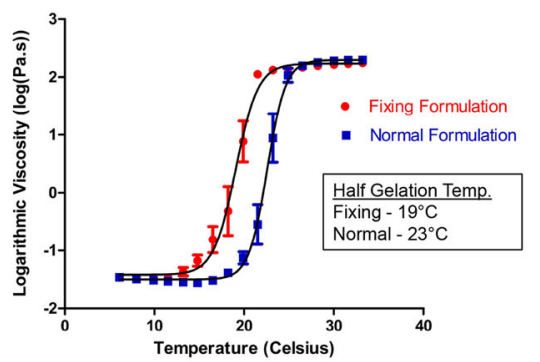

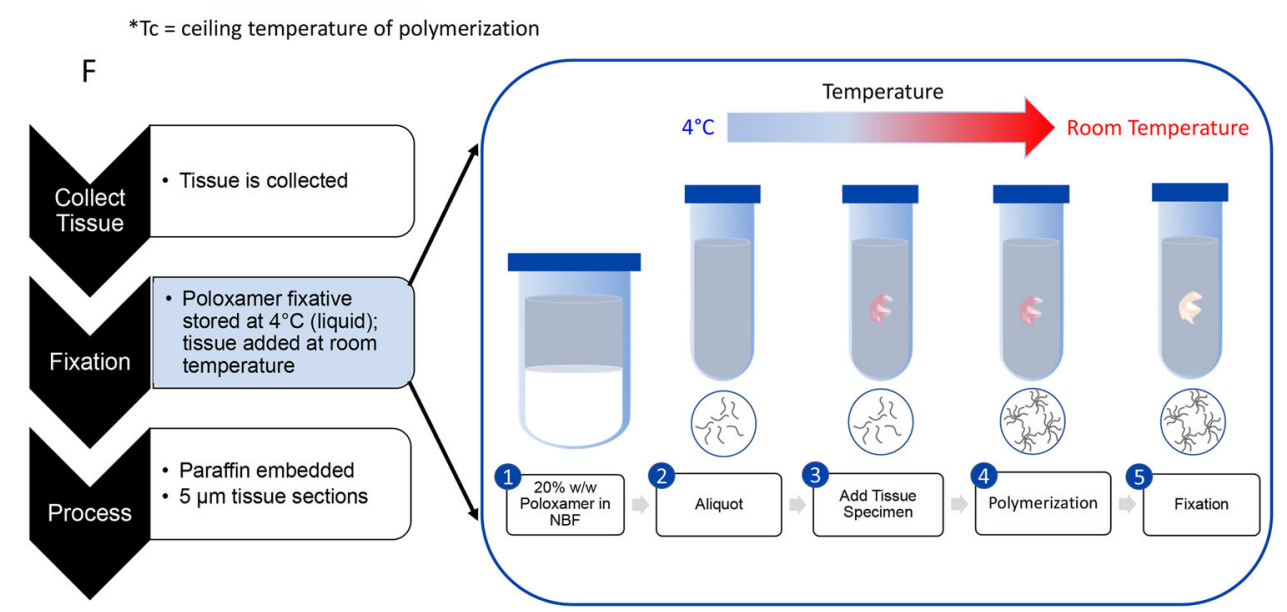

Fig. 2 Formulation of Poloxamer 407 fixative polymerizes at room temperature. The structural characteristics (a) of Poloxamer 407 (or Pluronic F-127) as an A-B-A block thermoreversible polymer (b) with the "A" blocks of this polymer being hydrophilic (blue) and the "B" block being hydrophobic (red). c Temperature increases to the ceiling temperature (Tc) enable oriented micelle formation with b block interactions towards the center of the micelle, while at temperatures beyond the Tc, hydrogen bonds between different micelles facilitate the formation of a polymer matrix. $\mathbf{d}$ The polymer matrix has similar physical properties and also forms non-covalent bonds with glyco-moieties of the highly glycosylated mucus matrix, allowing for efficient integration and scaffolding of Poloxamer 407 with the mucus layer. e Rheological testing to assess the polymer property of normal Poloxamer 407 (blue) and Poloxamer fixative (red) formulations as a function of temperature. Temperatures by which gelation is half maximum indicate a shift to a lower polymerization temperature comparing fixative versus normal formulations. f Histological workflow of tissue preparation with Poloxamer fixation. The fixation step in step 2 (e.g., with NBF) can be replaced with the Poloxamer fixative, while keeping the Poloxamer fixative on ice prior to addition to the tissue specimen. Fixation then undergoes the standard 24-h fixation period at room temperature (right).

transition into a gel state capable of providing scaffold support, we surmised that it would support the hydrophilic mucus architecture in histological applications.

For its adaptation into the clinical workflow, we formulated the Poloxamer 407 solution such that it had (1) fixation properties in addition to structural support, and (2) a lower ceiling temperature such that it can be used at room temperature in clinical suites. These properties enable scaffolded fixation of a specimen in a single step without additional equipment or processing steps. We formulated Poloxamer 407 to $20 \%(\mathrm{w} / \mathrm{w})$ in NBF solution to enhance mucus structure integrity during fixation and to initiate polymerization at room temperature for clinical specimen collection. Rheological assessment of Poloxamer 407 polymerization in water (normal formulation) versus formulation in NBF (hereafter, Poloxamer fixative) showed a shift in the polymerization temperature to room temperature (Fig. 2e). In a clinical workflow, Poloxamer fixative is stored at $4{ }^{\circ} \mathrm{C}$ prior to adding tissue at room temperature (Fig. 2f). The procedure listed was optimized to limit the introduction of extraneous variables and enhance ease of use in the clinic, and we have begun using Poloxamer fixative for limited human colonic biopsy specimen collection at Vanderbilt University Medical Center.
Poloxamer fixative enables in situ analysis of colonic microbes and host cells

To directly compare Carnoy's solution and Poloxamer fixative for histological applications, a portion of intact colonic tissue from each mouse was divided and fixed separately in each solution. We first tested IF antibodies that performed poorly in Carnoy's solution and Methacarn, and robust staining was observed with the Poloxamer fixative for PCNA and PCAD (Supplementary Fig. 1). To determine which fixation was best for visualizing both the mucus and epithelia, we performed serial multiplex FISH and IF on the same tissue sections using antibodies previously validated in Carnoy's solution. Five feature multiplex images (GOB5, Eub-eubacterial probe, PCK-pan-cytokeratin, WGA-wheat germ agglutinin, nuclei) are shown for the distal colon, where the highest density of microbes is found (Fig. 3). IF for PCK was used to delineate the epithelial layer (Fig. 3a, b, e, f, i, j). GOB5, also known as calciumactivated chloride channel regulator 1 (CLCA1), is a mucinprocessing protein that is secreted with mucus ${ }^{42}$. An intact mucus layer highlighted by antibody staining against GOB5 and WGA was observed with Carnoy's solution, Methacarn, and Poloxamer fixative (Fig. 3a, c, e, g, i, k). Notably, the Eub probe showed greater signal intensity with the Poloxamer fixative compared to Carnoy's solution 

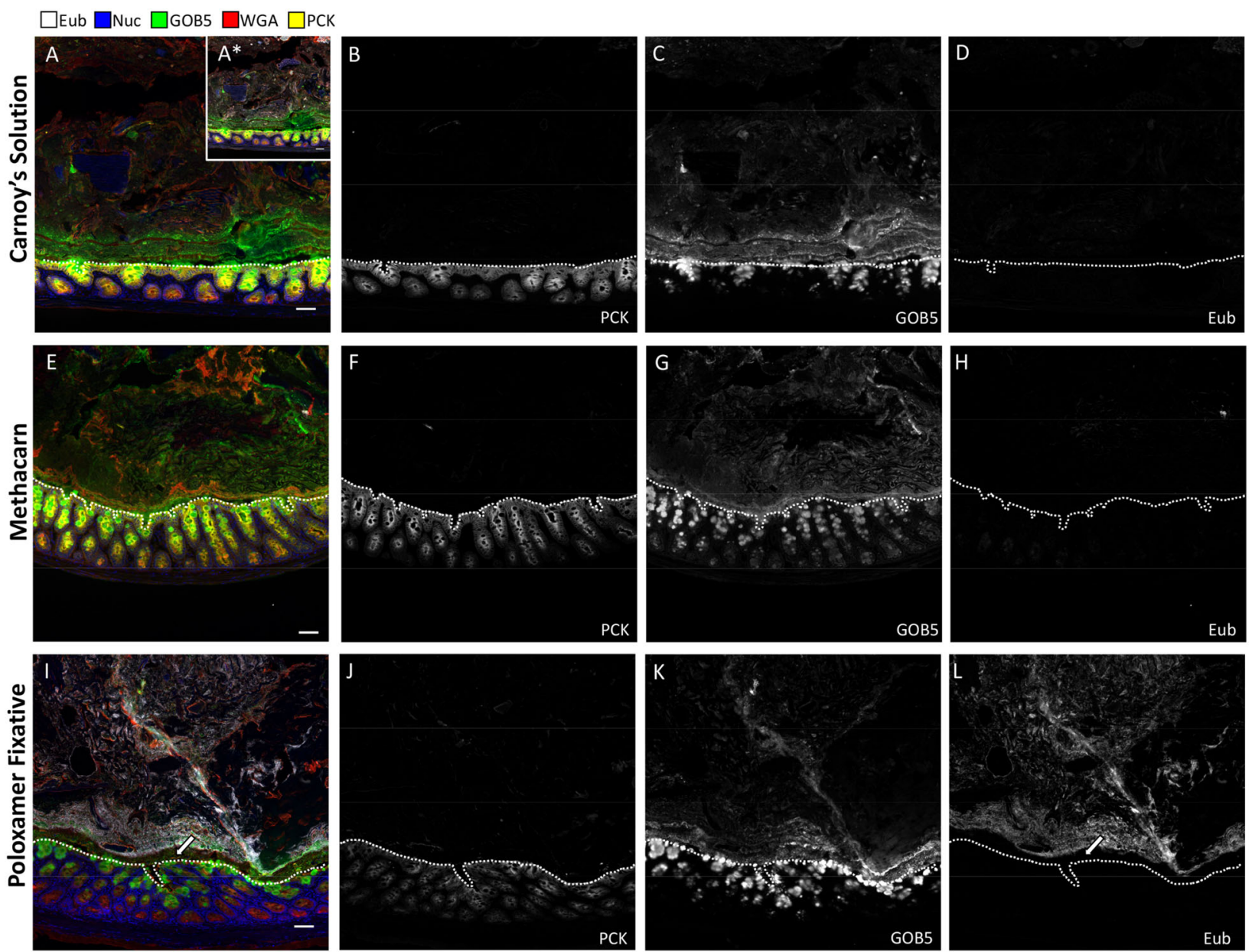

Fig. 3 Poloxamer fixation enables concurrent analysis of microbes and tissues, as compared with Carnoy's and Metharn fixation. IF of proteins coupled to bacterial FISH over multiple rounds of MxIF, with universal bacterial probe (Eub, white), nuclei (blue), GOB5 (green), lectin (WGA, red), and pan-cytokeratin (PCK, yellow). Selected individual channels (PCK, GOB5, Eub) to visualize epithelium, mucus, and bacteria, with the EUB probe having the same exposure times and gains to compare signal-to-noise in the two conditions. a-d Carnoy's solution, $\mathbf{e}-\mathbf{h}$ Methacarn, and i-I Poloxamer fixative were used for processing the same distal colonic tissue. $A^{*} \times 10$ gain was used to detect Eub probe in the Carnoy's condition. White dotted lines represent epithelial borders. Scale bars $=50 \mu \mathrm{m}$.

and Methacarn (Fig. 3a, d, e, h, i, I and Supplementary Fig. 2a). Bacteria were only observed with the Carnoy's solution after increased gain (Fig. 3a-inset), which exemplifies the challenges with biofilm detection reported with Carnoy's solution ${ }^{3,7,43}$. Consistent with previous studies ${ }^{6,8,11}$, the Eub signal is absent in the inner mucus layer closest to the epithelium (Fig. 3i, I, white arrow and Supplementary Fig. 2b). The thickness of the inner mucus layer, as denoted by the distance spanning this bacteria-free space, was not affected by different fixatives (Supplementary Fig. 2c). To evaluate mucus layer composition further, we stained Poloxamer and Methacarn-fixed colonic tissues with MUC2, UEA1, and WGA, the latter are lectins known to bind glycans on mucin. Mucus staining was consistent between the two fixatives, with strong, patterned staining of the inner mucus layer, and weaker, more heterogeneous, staining of the outer mucus layer (Supplementary Fig. 3). However, nonspecific, non-mucus cellular staining in the colonic lumen was observed in Methacarn, but not in Poloxamer fixative (Supplementary Fig. 3b, white arrow). These results demonstrate the improved sensitivity for concurrent FISH and IF imaging in colonic tissue with Poloxamer fixative.

Individual microbes are detected in Poloxamer-fixed tissue

To determine the specificity of the Eub probe with Poloxamer fixative, we tested distal colonic samples in parallel with a nonsense probe (Non-Eub). Low intensity staining was observed again with Carnoy's solution (Fig. 4a), which was further reduced to background level with the non-Eub probe (Fig. 4b). Poloxamerfixed tissue again had increased Eub staining sensitivity, and at higher magnification, individual, distinct rod-shaped bacteria were observed (Fig. 4c and inset). The non-Eub FISH probe also showed background level of signal in the Poloxamer-fixed tissue (Fig. 4d). These results demonstrate the enhanced sensitivity and specificity of FISH for detection of colonic microbiota with Poloxamer fixation.

To assess whether Poloxamer-fixed tissue can inform biologically relevant changes, we evaluated the mucus layer of dextran sulfate sodium (DSS)-treated animals. DSS induces localized damage to the colonic epithelium, resulting in a regenerative repair response. Regenerating epithelia initially lack glandular structure and are skewed towards proliferation instead of differentiation, resulting in a reduction of goblet cells ${ }^{44}$. Using the colonic architecture and goblet cell numbers as markers, we concurrently observed damaged and normal-appearing regions within the same mouse colon (Supplementary Fig. 4A, B). The inner mucus layer overlying the damaged regions appeared to be thinner compared to that of normal regions, with the epithelium in closer proximity to the microbiome as marked by the Eub probe (Supplementary Fig. 4). Furthermore, Eub probe signal appeared in the crypts of damaged areas (Supplementary Fig. 4A, B), a 

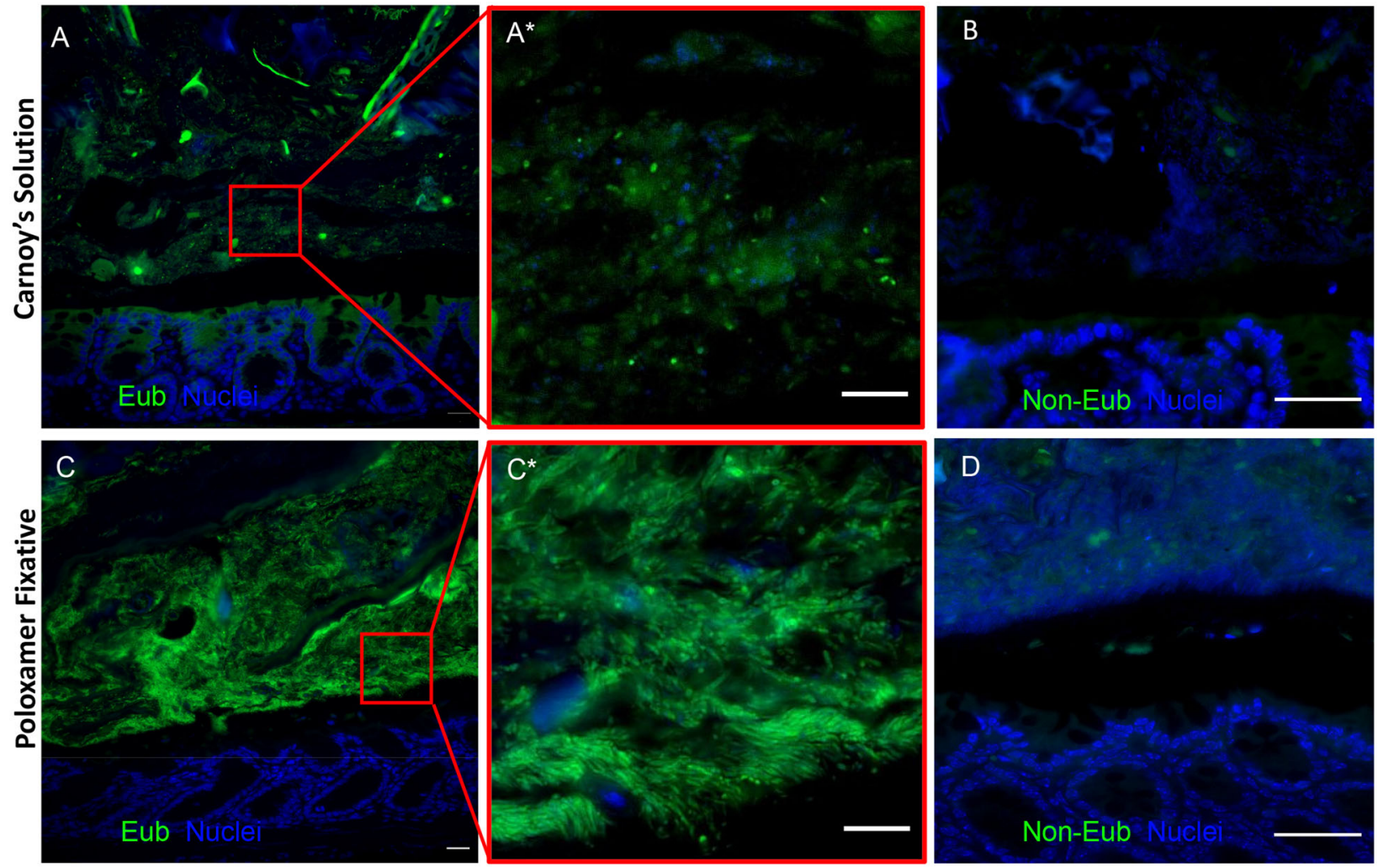

Fig. 4 Specific, individual bacteria are identified in Poloxamer-fixed tissue. FISH images of mouse distal colon. a Carnoy's-fixed colon stained with the Eub (green) probe, and $\mathbf{b}$ non-Eub probe. $\mathbf{c}$ Poloxamer-fixed colon stained with the Eub (green) probe, and $\mathbf{d}$ non-Eub probe. $A^{*}$ and $C^{*}$ are higher magnification views of red insets in $\mathbf{a}, \mathbf{c}$. Scale bars $(\mathbf{a}, \mathbf{c})=20 \mu \mathrm{m},\left(A^{*}, \mathbf{b}, C^{*}, \mathbf{d}\right)=10 \mu \mathrm{m}$.

phenomenon not observed in normal regions or colons of healthy mice. The reduction in mucus is consistent with the decrease in goblet cells induced by DSS damage, and microbial encroachment into crypts may form the basis of inflammatory interactions with the mucosa.

Mucosa-associated biofilms are observed in colonic tissue using Poloxamer fixation

To further validate Poloxamer fixative for biofilm detection with direct implications for the human microbiome, we colonized germ-free (GF) mice with a biofilm-positive microbiota slurry isolated from a human colorectal cancer (Fig. 5a) ${ }^{15}$. With Poloxamer fixation, we were able to observe bacteria forming dense colonies in direct contact with the colonic epithelium, without an inner mucus layer that typically separates luminal bacteria from the epithelium (Fig. 5b). For our negative control, we observed that no bacteria were detected in sham-inoculated mice, although a mucus layer was maintained (Fig. 5c). Multiplex FISH for Lachnospiraceae, Bacteroidetes (CFB), Fusobacteria, and Gammaproteobacteria performed on the same tissue section demonstrated spatially heterogeneous patterns of colonization (Fig. $5 \mathrm{~d}$ ). For example, we observed areas that are rich in Bacteroidetes with CFB staining (Fig. 5d-inset), while adjacent colonies are enriched for Gammaproteobacteria but devoid of CFB (Fig. $5 d$, white arrow). Fusobacteria were not detected by FISH, consistent with previous 16S rRNA sequencing at 1 week post inoculation (Fig. 5e $)^{15}$. Multiplex FISH and IF revealed that more F4/80+ cells (macrophages) infiltrated into the mucosa adjacent to colonies established by the slurry, but not further away, demonstrating a distinct host response dependent on spatial proximity (Supplementary Fig. 5). These results demonstrate that Poloxamer fixative enables spatial analysis of mucosa-associated biofilms and host biology by multiplex FISH and IF.
Poloxamer fixation permits detection of mucosa-associated biofilms in human colonic adenomas

To demonstrate the clinical utility of our method, portions of human colonic adenomas collected during colonoscopy were immediately placed into Poloxamer fixation for subsequent FISHbased biofilm analysis (Fig. 2f and Supplementary Fig. 6). Using a scoring scheme defined previously ${ }^{3,12,13,15}$, biofilm detection (>2.5 score) was consistent in Poloxamer fixative compared to prior methods (Table 1). Mucosa-associated biofilms were detected on adenomas arising from the ascending colon, compared with ascending normal colons from the same patient which lack biofilm (Fig. 6). Bacterial invasion into the adenoma was observed in one polyp (Fig. 6e, white arrows). The advantages of existing repertoires of antibodies and effective bacterial FISH staining enabled robust screening for biofilms in human specimens fixed in the Poloxamer fixative, suggesting that this approach can be a useful clinical tool.

\section{DISCUSSION}

Microbiota-host interactions are implicated in a range of disorders, including CRC. Specifically, human CRC-associated stool microbiota transplanted into GF or carcinogen-treated mice exacerbates colon epithelial cell proliferation and the tumor-associated immune response ${ }^{45}$. Colonic mucosa-associated biofilms are defined by the direct interaction between bacteria and epithelial cells. They are present in $\sim 89 \%$ and $12 \%$ of right-sided and left-sided colon tumors, respectively ${ }^{3}$. CRC in human FAP appears to be uniformly biofilm positive ${ }^{13}$. Some human colonic biofilms are carcinogenic in GF ApcMin mice ${ }^{15}$. With a reported range of $13-35 \%$ colonic biofilm positive in healthy subjects, these findings indicate clinical relevance for using biofilm to stratify high-risk precancer lesions.

The established tissue fixation for detecting colonic biofilms is Carnoy's solution. A major limitation of Carnoy's solution is that IF 

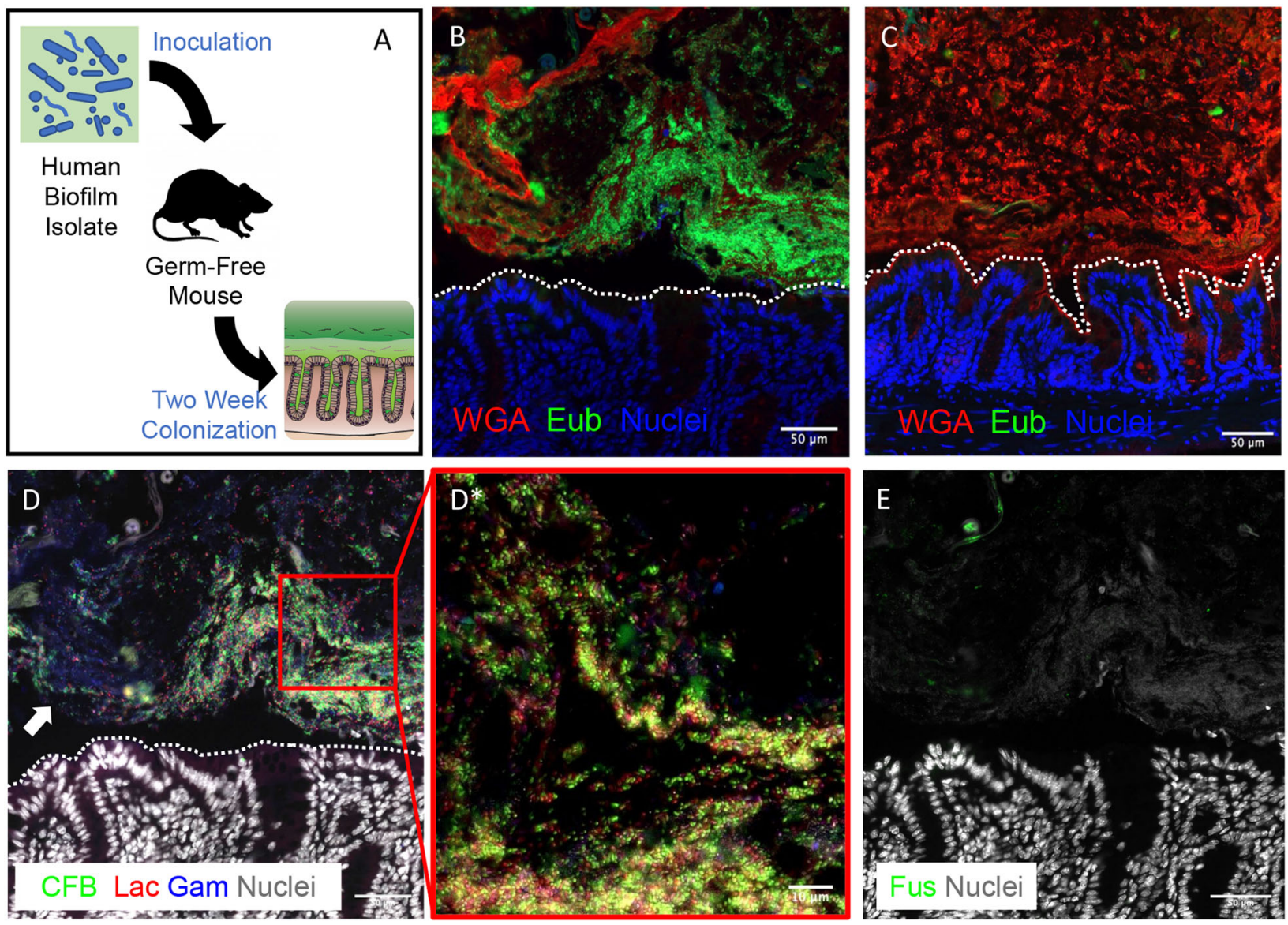

Fig. 5 Mouse colons colonized with human CRC patient mucosal homogenate forms mucosa-associated biofilms. a A schematic of germfree (GF) mouse inoculation with human biofilm-positive mucosal slurries. Mice were sacrificed 2 weeks post inoculation and processed using the Poloxamer fixative. Imaging was performed using WGA (red) for mucus, Eub (green) for bacteria, and nuclei (blue) under different conditions of inoculation into GF mice: $\mathbf{b}$ Human CRC biofilm isolate, and $\mathbf{c}$ sham. $\mathbf{d}$ Iterative FISH staining rounds with Bacteroidetes (green), Lachnospiraceae (red), and Gammaproteobacteria (blue), e Fusobacteria (green) probes of the tissue in b. Scale bars $=50 \mu \mathrm{m}$. White dotted lines represent epithelial borders. $\mathbf{d}^{*}$ is a higher magnification view of red inset in $\mathbf{d}$. Scale bars $=10 \mu \mathrm{m}$.

Table 1. Biofilm characteristics of human specimens.

\begin{tabular}{|c|c|c|c|c|c|}
\hline Sample ID & $\begin{array}{l}\text { Mucus } \\
\text { present }^{\mathrm{a}}\end{array}$ & $\begin{array}{l}\text { Bacteria abundance } \\
\text { per } 200 \mu \mathrm{m} \mathrm{CEC}{ }^{b}\end{array}$ & $\begin{array}{l}\text { Percentage of } \\
\text { biofilm coverage }^{c}\end{array}$ & $\begin{array}{l}\text { Is there DAPI staining } \\
\text { without Cy3? }\end{array}$ & Notes \\
\hline $\begin{array}{l}\text { MPP00048A1 } \\
\text { HTA11_1429 }\end{array}$ & 0.5 & 3 & $50 \%$ & No & $\begin{array}{l}\text { Mostly rods, some cocci in BF; not great mucus } \\
\text { though; some autofluorescent objects (larger } \\
\text { than bacteria) to watch out for in some regions }\end{array}$ \\
\hline $\begin{array}{l}\text { MPP00049A1 } \\
\text { HTA11_1391 }\end{array}$ & 1 & 3 & $5 \%$ & No & $\begin{array}{l}\text { Lots of nice rods everywhere, BF region located } \\
\text { within crypts of CEC in one area }\end{array}$ \\
\hline
\end{tabular}

sensitivity is reduced and inadequate for detecting epithelial antigens (Fig. 1). Generally, dehydration fixation approaches are able to retain mucus in samples, but they also reduce the efficacy of FISH staining ${ }^{11,46}$. Conventional FFPE tissue is limited by the poor preservation of the mucus layer (Fig. 1). Our goal was to develop a broadly applicable reagent that preserves mucus and biofilms without compromising IF detection of host factors. With the Poloxamer fixative, we overcame the need to apply heat for polymerization and developed a clinically compatible, room temperature reagent (Fig. 2).

We demonstrated how Poloxamer-fixed samples were superior to Carnoy's-fixed samples for visualizing both mucosa-associated bacteria and host cells in the same tissue section (Fig. 3). Whereas agarose has been shown to also provide physical scaffold ${ }^{36}$, the physical and chemical properties of Poloxamer make it the ideal substrate for preservation of mucus. The Poloxamer fixative polymerizes at room temperature, enabling its incorporation into existing clinical protocols for human studies. Methods that enable multimodal analysis will become very valuable in future endeavors for understanding complex tissue ecosystems. Our efforts represent an advance in the field of multiplex imaging, and one of the first to simultaneously evaluate composition and organization of both microbes and host cells. 


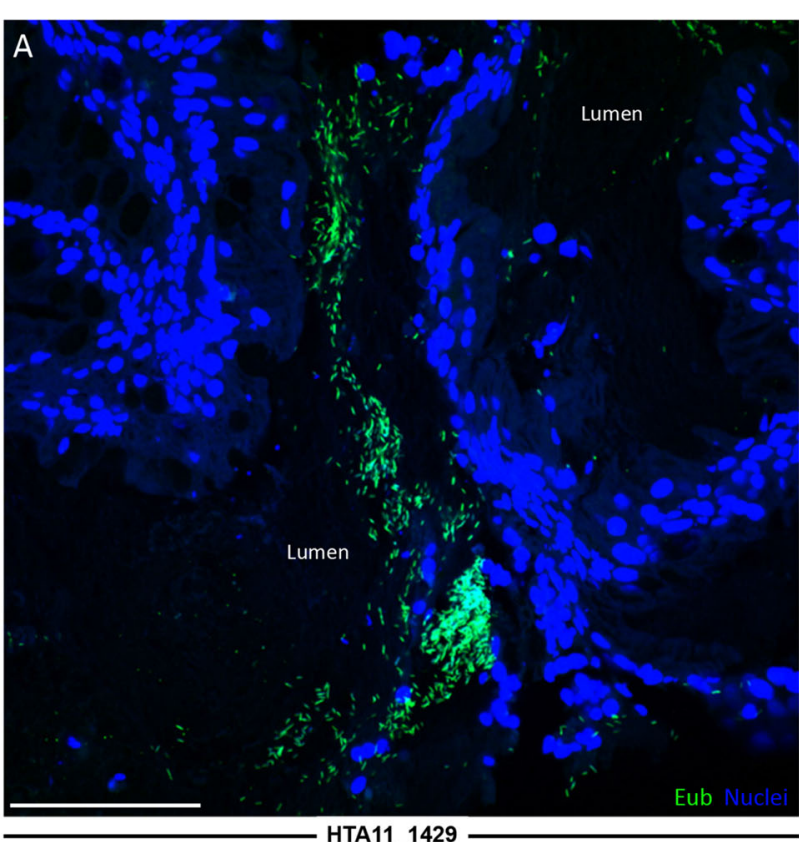

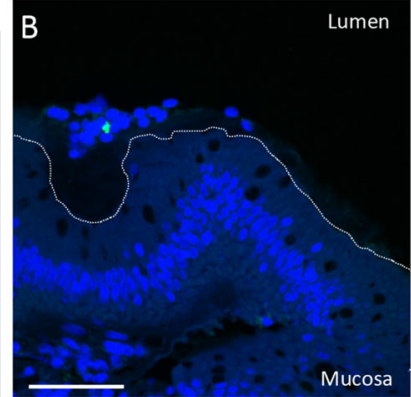

Asc. Colon for HTA11 1429

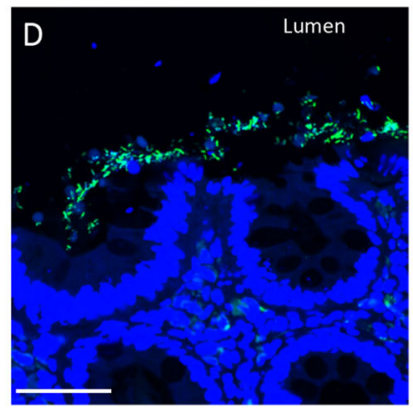

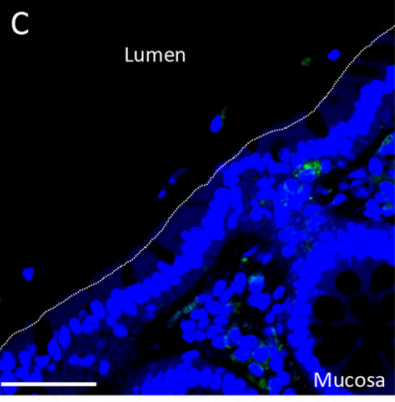

Asc. Colon for HTA11_1391

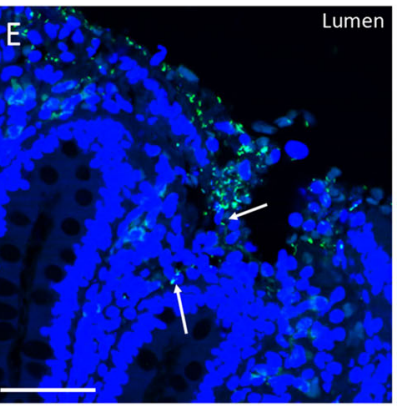

HTA11_1391

Fig. 6 Biofilm-positive human colonic polyps demonstrate invasion of bacteria into the mucosa. $\times 63$ confocal images of human colonic tissues fixed in Poloxamer showing regions of biofilm positivity using Eub (green) probes to identify bacteria. a Patient polyp sample HTA11_1429_20_2013 with (b) normal ascending colon. c Normal ascending colon with (d, e) patient polyp sample HTA11_1391_20_1011. White arrows indicate invaded bacteria. White dotted lines represent epithelial borders in normal colon. Scale bars $=50 \mu \mathrm{m}$.

Previous assessments of biofilms relied on consensus of experts to score samples for determining biofilm positivity ${ }^{3,12,13}$. The increased FISH signal observed with Poloxamer fixative potentially enables the identification of biofilms with improved technical proficiency and machine learning. Unlike sequencing approaches to characterize the microbiome, FISH requires preselection of candidates, but it provides spatial information important for determining microbial behaviors ${ }^{7,14}$.

To our knowledge, this is the first use of Poloxamer 407 for tissue fixation $37,39,40$. Our application of Poloxamer fixation is directed towards room temperature, standard histological applications. Substitution of Poloxamer for OCT as a scaffold for frozen tissue application has not been successful at this time due to the gelation point of Poloxamer at about $19^{\circ} \mathrm{C}$. Nevertheless, our successful implementation of this fixative to conventional histological pipelines will enable its wider deployment in clinical pipelines for human microbiome and biofilm research.

\section{METHODS}

Tissue fixation and processing

For Methacarn fixation, tissues were immersed in Methacarn solution (6:3:1 ratio of methanol:glacial acetic acid:chloroform) and processed using standard procedure ${ }^{6}$. A similar procedure was followed for Carnoy's solution fixation with the exception of the use of ethanol (6:3:1 ratio of ethanol:glacial acetic acid:chloroform). Tissues were also fixed using standard NBF fixation. For Poloxamer fixation, the Poloxamer 407 solution was made at $4{ }^{\circ} \mathrm{C}$, where $20 \% \mathrm{w} / \mathrm{w}$ Poloxamer was mixed into $10 \% \mathrm{NBF}$. Aliquots were kept on ice prior to the addition of the tissue specimen. Once the specimen was immersed in the solution, the mixture was brought to room temperature at $25^{\circ} \mathrm{C}$ and allowed to polymerize. The mixture was visually inspected for polymerization by checking for the reduction of movement when the conical tube was inverted. From here, the tissue was fixed at room temperature for 24-h, followed by standard histological processing. Tissue blocks prepared from fixed tissues were sectioned at 5 microns onto slides ${ }^{22}$.

\section{Rheological testing}

To test for polymer gelation properties, rheological testing was performed using Rheometer AR2000 ex machine with both the Poloxamer fixative formulation ( $20 \% \mathrm{w} / \mathrm{w}$ Poloxamer 407 into $10 \% \mathrm{NBF})$ and normal Poloxamer formulation ( $20 \% \mathrm{w} / \mathrm{w}$ Poloxamer 407 into water). To test sol-gel transitions, temperature was modulated from $5{ }^{\circ} \mathrm{C}$ to $40^{\circ} \mathrm{C}$ with controlled stress and rate $e^{38,40}$, and half gelation temperature was calculated by a dose response non-linear log fit in Prism (Graphpad).

\section{Tissue collection}

Conventional mouse experiments were performed under protocols approved by the Vanderbilt University Animal Care and Use Committee and in accordance with $\mathrm{NIH}$ guidelines. Animals were euthanized and colonic tissues were collected using previously published procedures 47,48 . GF mouse experiments were performed at Johns Hopkins University under protocols approved by the Johns Hopkins University Animal Care and Use Committee in accordance with $\mathrm{NIH}$ guidelines.

Human colonic tissues were collected from participants in the Colorectal Molecular Atlas Project (COLON MAP), a study approved by Vanderbilt Institutional Review Board. All participants provided written informed consent. Participants underwent colonoscopy as part of their routine care following standard bowel cleansing preparation. Polyps were removed according to standard of care and bisected. Visually normal mucosal biopsies were obtained in the mid-ascending colon using jumbo biopsy forceps. To increase likelihood of biofilm positivity, only participants with a polyp in the cecum or ascending colon were included in this analysis. Each tissue (either normal biopsy or polyp portion) was placed in individual labeled tubes of Poloxamer fixative, and gently inverted twice to ensure full coverage of the sample.

\section{Multiplex fluorescence imaging}

Sequential antibody staining and dye inactivation was performed as described $^{22}$. FISH probes, antibodies, and lectins used are listed in Tables 2 and 3. Modifications to the protocol includes the incorporation of FISH, as described below, prior to IF. Fluorescence of FISH probes was inactivated using the same methods as antibodies. For direct comparisons, tissues were imaged with the same exposure time using an Olympus X81 inverted microscope with a motorized stage with filter sets for DAPI, GFP, CY3, CY5, and CY7. Images were also collected on a Zeiss Axio-Imager M2 microscope. 
Table 2. Antibodies and lectins used in this study.

\begin{tabular}{lllllllc}
\hline Marker & Channel & Exposure & Concentration & Product ID & Clone/Cat \# & Clonality & Host species \\
\hline PCK & FITC & $100 \mathrm{~ms}$ & $1: 200$ & ab11214 & PCK-26 & Monoclonal & Mouse \\
GOB5 & 647 & $100 \mathrm{~ms}$ & $1: 200$ & ab180851 & EPR12254-88 & Monoclonal & Rabbit \\
PCNA & 488 & $200 \mathrm{~ms}$ & $1: 100$ & CS 85805 & P10 & Monoclonal & Mouse \\
PCAD & Cy3 & $100 \mathrm{~ms}$ & $1: 100$ & DC13090 & RB-9036 & Polyclonal & Rabbit \\
F4/80 & Cy5 & $1000 \mathrm{~ms}$ & $1: 200$ & BL123115 & BM8 & Monoclonal & Rat \\
MUC2 & Cy5 & $1000 \mathrm{~ms}$ & $1: 100$ & sc-15334 & H-300 & Polyclonal & Rabbit \\
WGA & $488 / C y 5$ & $20 \mathrm{~ms}$ & $1: 100$ & W7024, W32466 & NA & NA & NA \\
UEA 1 & Cy5 & $10 \mathrm{~ms}$ & $1: 1000$ & B-1065 & NA & NA \\
\hline
\end{tabular}

Table 3. Nucleic acid probes used in this study.

\begin{tabular}{lllll}
\hline Probe & Probe sequence & Exposure & Concentration \\
\hline Eub & 5'-Cy3-GCTGCCTCCCGTAGGAGT-3' $^{\prime}$ & $100 \mathrm{~ms}$ & $1: 100$ & Label \\
CFB286 & 5'-Fluorescein-TCCTCTCAGAACCCCTAC-3' $^{\prime}$ & $150 \mathrm{~ms}$ & $1: 100$ & Mast bacteria \\
FUS714 & 5'-Cy7-GGCTTCCCCATCGGCATT-3' $^{\prime}$ & $200 \mathrm{~ms}$ & $1: 100$ & Fusobacterium \\
Lac435 & 5'-Cy3-TCTTCCCTGCTGATAGA-3' $^{\prime}$ & $100 \mathrm{~ms}$ & $1: 100$ & Lachnospiraceae \\
Gam42a & 5'-Cy5-GCCTTCCCACATCGTTT-3' $^{\prime}$ & $500 \mathrm{~ms}$ & $1: 100$ & Gammaproteobacteria \\
Non-Eub & 5'-Cy3-CGACGGAGGGCATCCTCA-3' $^{\prime}$ & $200 \mathrm{~ms}$ & $1: 100$ & Nonsense probe \\
\hline
\end{tabular}

Fluorescent in situ hybridization

FISH probes were suspended in sterile water at a concentration of $0.2 \mathrm{nmol} / \mu \mathrm{l}(200 \mu \mathrm{M})$ and stored at $-20^{\circ} \mathrm{C}$. Hybridization buffer $(20 \mathrm{mM}$ Tris- $\mathrm{HCl}$ [pH 8.0], $0.9 \mathrm{M} \mathrm{NaCl}, 0.01 \%$ sodium dodecyl sulfate) and FISH wash buffer $\left(225 \mathrm{mM} \mathrm{NaCl}, 20 \mathrm{mM}\right.$ Tris, $5 \mathrm{mM}$ EDTA) were pre-warmed in a $46^{\circ} \mathrm{C}$ oven. Slides were then de-paraffinized through $3 \times 5$ min dips in Histoclear under the fume hood. Re-hydration of slides was done using an ethanol gradient. Slides were placed for $5 \mathrm{~min}$ in each of the following solutions: $100 \% \mathrm{ETOH}, 100 \% \mathrm{ETOH}, 95 \% \mathrm{ETOH}, 95 \% \mathrm{ETOH}, 70 \% \mathrm{ETOH}$, followed by storage in $20 \mathrm{mM}$ Tris buffer. Probes were diluted to $2 \mu \mathrm{M}$ in hybridization buffer prior to application. Approximately $100 \mu \mathrm{l}$ volume was applied per sample to fully cover the fixed tissue. Slides were then incubated for $\sim 1.5-2 \mathrm{~h}$ at $46^{\circ} \mathrm{C}$ in a humidified chamber $(1.5 \mathrm{~h}$ for the universal probe; $2 \mathrm{~h}$ for specific taxa probes). Slides were washed with FISH wash buffer $3 \times 5$ min on shaker.

Quantitative image analysis

For the host mucosa, binary mucosal masks were generated through DAPI channel thresholding to create regions of interest limited to the mucosa and not lumen. For the colonic lumen, binary luminal masks were generated through thresholding EUB expression and the elimination of the mucosal mask. For intensity quantifications, mean fluorescent intensities were obtained for FISH or antibody staining within the respective binary masks. For calculating inner mucus layer thickness, the outer edges of the mucosal and luminal masks were identified. The minimal distances between the two edges were calculated iteratively along their lengths by sampling 100 random points along the luminal edge. All image analyses were performed using MATLAB (MathWorks).

\section{Biofilm screening}

Patient tissues were collected and screened for biofilms using the universal bacterial probe (EUB338), as described previously ${ }^{3,12,13}$. Briefly, tissues were defined as biofilm positive if there were more than $10^{9}$ bacteria/ml invading the mucus layer (within $1 \mu \mathrm{m}$ of the epithelium) for at least $200 \mu \mathrm{m}$ of the epithelial surface.

\section{Human tissue inocula preparation}

A sample was prepared from a 3-mm diameter tissue piece collected from a resected $\mathrm{CRC}$, snap-frozen, and stored at $-80^{\circ} \mathrm{C}$. The inoculum was prepared anaerobically by mincing and homogenizing tissue pieces in PBS in an anaerobic hood to a dilution of 1:20 (weight/volume). Prior to gavage into GF mice, the inoculum was further diluted 1:10 in PBS, for a final of $1: 200 \mathrm{w} / \mathrm{v}$ dilution from the original tissue.

\section{Mouse colonization}

GF C57BL/6 wild-type animals were transferred to gnotobiotic isolators (separate isolator for each experimental group) and gavaged with $100 \mu \mathrm{l}$ of the human inoculum. Mice were euthanized at indicated time points ${ }^{15}$. About 1 to $2 \times 0.5 \mathrm{~cm}$ snips were taken from the proximal and distal colon, and then fixed in accordance to the protocols above. Tissues were processed, paraffin-embedded, and sectioned.

\section{DSS administration}

Conventionally housed C57BL/6 wild-type animals were administered $2.5 \%$ DSS in drinking water for the following 6 days, prior to a 9-day rest period, followed by a second round of DSS. Tissues were collected at the end of the second DSS round.

\section{Reporting summary}

Further information on research design is available in the Nature Research Reporting Summary linked to this article.

\section{DATA AVAILABILITY}

All data generated or analyzed during this study are included in this published article (and its supplementary information files). Additional imaging data are available from the corresponding author on reasonable request.

Received: 31 March 2020; Accepted: 28 August 2020;

Published online: 24 September 2020

\section{REFERENCES}

1. Feng, Q. et al. Gut microbiome development along the colorectal adenoma-carcinoma sequence. Nat. Commun. 6, 6528 (2015).

2. Baxter, N. T., Zackular, J. P., Chen, G. Y. \& Schloss, P. D. Structure of the gut microbiome following colonization with human feces determines colonic tumor burden. Microbiome 2, 20 (2014). 
3. Dejea, C. M. A. W. E. C. et al. Microbiota organization is a distinct feature of proximal colorectal cancers. Proc. Natl Acad. Sci. 111, 18321-18326 (2014).

4. Drewes, J. L. et al. High-resolution bacterial $16 \mathrm{~S}$ rRNA gene profile meta-analysis and biofilm status reveal common colorectal cancer consortia. NPJ Biofilms Microbiomes 3, 34 (2017).

5. Chen, J., Domingue, J. C. \& Sears, C. L. Microbiota dysbiosis in select human cancers: evidence of association and causality. Semin. Immunol. 34, 25-34 (2017).

6. Johansson, M. E. et al. The inner of the two Muc2 mucin-dependent mucus layers in colon is devoid of bacteria. Proc. Natl Acad. Sci. 105, 15064-15069 (2008).

7. Earle, K. A. et al. Quantitative imaging of gut microbiota spatial organization. Cell Host Microbe 18, 478-488 (2015).

8. Donaldson, G. P., Lee, S. M. \& Mazmanian, S. K. Gut biogeography of the bacterial microbiota. Nat. Rev. Microbiol. 14, 20-32 (2015).

9. Johansson, M. E., \& Hansson, G. C. Preservation of mucus in histological sections, immunostaining of mucins in fixed tissue, and localization of bacteria with FISH. Methods Mol. Biol. 842, 229-235 (2012).

10. Lavelle, A. et al. Spatial variation of the colonic microbiota in patients with ulcerative colitis and control volunteers. Gut 64, 1553-1561 (2015).

11. Tropini, C., Earle, K. A., Huang, K. C. \& Sonnenburg, J. L. The gut microbiome: connecting spatial organization to function. Cell Host Microbe 21, 433-442 (2017)

12. Drewes, J. L. et al. High-resolution bacterial $16 \mathrm{~S}$ rRNA gene profile meta-analysis and biofilm status reveal common colorectal cancer consortia. npj Biofilms Microbiomes 3, 34 (2017).

13. Dejea, C. M. et al. Patients with familial adenomatous polyposis harbor colonic biofilms containing tumorigenic bacteria. Science 359, 592-597 (2018).

14. Swidsinski, A., Weber, J., Loening-Baucke, V., Hale, L. P. \& Lochs, H. Spatia organization and composition of the mucosal flora in patients with inflammatory bowel disease. J. Clin. Microbiol. 43, 3380-3389 (2005).

15. Tomkovich, S., et al. Human colon mucosal biofilms from healthy or colon cancer hosts are carcinogenic. J Clin. investig. 129, 1699-1712 (2019).

16. Costerton, J. W., Stewart, P. S. \& Greenberg, E. P. Bacterial biofilms: a common cause of persistent infections. Science 284, 1318-1322 (1999).

17. Mallick, $\mathrm{H}$. et al. Predictive metabolomic profiling of microbial communities using amplicon or metagenomic sequences. Nat. Commun. 10, 1-11 (2019).

18. Chen, K. H., Boettiger, A. N., Moffitt, J. R., Wang, S. \& Zhuang, X. Spatially resolved, highly multiplexed RNA profiling in single cells. Science 348, aaa6090 (2015).

19. Gerdes, M. J. et al. Highly multiplexed single-cell analysis of formalin-fixed, paraffin-embedded cancer tissue. Proc. Natl Acad. Sci. 110, 11982-11987 (2013).

20. Eng, C. H. L. et al. Transcriptome-scale super-resolved imaging in tissues by RNA seqFISH. Nature 568, 235-239 (2019).

21. Goltsev, Y. et al. Deep profiling of mouse splenic architecture with CODEX multiplexed imaging. Cell 174, 968-981 (2018).

22. McKinley, E. T., et al. Optimized multiplex immunofluorescence single-cell analysis reveals tuft cell heterogeneity. JCI Insight 2, e93487 (2017).

23. Lin, J. R., Fallahi-Sichani, M. \& Sorger, P. K. Highly multiplexed imaging of single cells using a high-throughput cyclic immunofluorescence method. Nat. Commun. 6, 8390 (2015).

24. Tjalsma, H., Boleij, A., Marchesi, J. R. \& Dutilh, B. E. A bacterial driver-passenger model for colorectal cancer: beyond the usual suspects. Nat. Rev. Microbiol. 10, 575-582 (2012)

25. Gut, G., Herrmann, M. D. \& Pelkmans, L. Multiplexed protein maps link subcellular organization to cellular states. Science 361, eaar7042 (2018).

26. Remark, R. et al. In-depth tissue profiling using multiplexed immunohistochem ical consecutive staining on single slide. Sci. Immunol. 1, aaf6925 (2016)

27. Jungmann, R. et al. Multiplexed 3D cellular super-resolution imaging with DNAPAINT and exchange-PAINT. Nat. Methods 11, 313-318 (2014).

28. Biedermann, L. \& Rogler, G. The intestinal microbiota: its role in health and disease. Eur. J. pediatrics 174, 151-167 (2015).

29. Abraham, C. \& Medzhitov, R. Interactions between the host innate immune system and microbes in inflammatory bowel disease. Gastroenterology $\mathbf{1 4 0}$ 1729-1737 (2011).

30. Bromberg, L. E. \& Barr, D. P. Self-association of mucin. Biomacromolecules 1 , 325-334 (2000).

31. Atuma, C., Strugala, V., Allen, A., \& Holm, L. The adherent gastrointestinal mucus gel layer: thickness and physical state in vivo. J. Am. Physiol. Soc. 280, G922-G929 (2001).

32. Rizzardi, A. E. et al. Quantitative comparison of immunohistochemical staining measured by digital image analysis versus pathologist visual scoring. Diagnostic Pathol. 7, 42 (2012).

33. Robertson, D., Savage, K., Reis-Filho, J. S. \& Isacke, C. M. Multiple immunofluorescence labelling of formalin-fixed paraffin-embedded (FFPE) tissue. BMC cell Biol. 9, 13 (2008)

34. Dongari-Bagtzoglou, A. Pathogenesis of mucosal biofilm infections: challenges and progress. Expert Rev. Anti Infect. Ther. 6, 201-208 (2008).
35. Demouveaux, B., Gouyer, V., Gottrand, F., Narita, T. \& Desseyn, J. L. Gel-forming mucin interactome drives mucus viscoelasticity. Adv. Colloid Interface Sci. 252, 69-82 (2017).

36. Hasegawa, Y., Welch, J. L. M., Rossetti, B. J. \& Borisy, G. G. Preservation of threedimensional spatial structure in the gut microbiome. PLOS ONE 12, e0188257 (2017).

37. Dumortier, G., Grossiord, J. L., Agnely, F. \& Chaumeil, J. C. A review of poloxamer 407 pharmaceutical and pharmacological characteristics. Pharm. Res. 23, 2709-2272 (2006).

38. Charrueau, C., Tuleu, C., Astre, V., Grossiord, J. L. \& Chaumeil, J. C. Poloxamer 407 as a thermogelling and adhesive polymer for rectal administration of short-chain fatty acids. Drug Dev. Ind. Pharm. 27, 351-357 (2001).

39. Giuliano, E., Paolino, D., Fresta, M. \& Cosco, D. Mucosal applications of poloxamer 407-based hydrogels: an overview. Pharmaceutics 10, 159 (2018).

40. Baloglu, E., Karavana, S. Y., Senyigit, Z. A., \& Guneri, T. Rheological and mechanical properties of poloxamer mixtures as a mucoadhesive gel base. Pharm. Dev. Technol. 16, 627-636 (2011).

41. Fakhari, A., Corcoran, M. \& Schwarz, A. Thermogelling properties of purified poloxamer 407. Heliyon 3, e00390 (2017).

42. Nyström, E. E., Arike, L., Ehrencrona, E., Hansson, G. C. \& Johansson, M. E. Calciumactivated chloride channel regulator 1 (CLCA1) forms non-covalent oligomers in colonic mucus and has mucin 2-processing properties. J. Biol. Chem. 294 17075-17089 (2019).

43. Arena, E. T., et al. Bioimage analysis of Shigella infection reveals targeting of colonic crypts. Proc. Natl Acad. Sci. 112, E3282-E3290 (2015).

44. Nowarski, R. et al. Epithelial IL-18 equilibrium controls barrier function in colitis. Cell 163, 1444-1456 (2015).

45. Wong, S. H. et al. Gavage of fecal samples from patients with colorectal cancer promotes intestinal carcinogenesis in germ-free and conventional mice. Gastroenterology 153, 1621-1633 (2017).

46. Urieli-Shoval, S. et al. Preservation of RNA for in situ hybridization: Carnoy's versus formaldehyde fixation. J. Histochemistry Cytochemistry 40, 1879-1885 (1992).

47. Liu, Q. et al. Quantitative assessment of cell population diversity in single-cell landscapes. PLoS Biol. 16, e2006687 (2018).

48. Simmons, A. J., et al. Cytometry-based single-cell analysis of intact epithelial signaling reveals MAPK activation divergent from TNF-a-induced apoptosis in vivo. Mol. Syst. Biol. 11, 835 (2015).

\section{ACKNOWLEDGEMENTS}

The authors would like to thank Dr. Cynthia Reinhart-King for advice on polymers, Katarzyna Zienkiewicz and Dr. Scott Guelcher for rheometer use, and Dr. Eliot McKinley for his advice on MxIF use. K.S.L. and A.J.S. are funded by R01DK103831. R.J. C. is funded by P50CA236733 and R35CA1975703. M.C.M., J.L.D., J.T.R., R.J.C., M.J.S., C.L.S., and K.S.L. are funded by U2CCA233291. N.O.M. is funded by T32DK007673. P.N V. is funded by T32HD007502. C.R.S. is funded T32Al007281. Participant recruitment and biospecimen collection were conducted in part by the Survey and Biospecimen Shared Resource which is supported in part by P30CA68485. Tissue fixation and processing was provided by the $\mathrm{NCl}$ Cooperative Human Tissue Network (CHTN) Western Division UM1CA183727.

\section{AUTHOR CONTRIBUTIONS}

M.C.M. conceived the study, performed experiments, analyzed the data, compiled the figures, and wrote the manuscript. J.L.D. performed microbiome transplant experiments, scored human specimens, and contributed to the writing of the manuscript. N.O.M. assisted with mouse experiments, intellectually contributed to the study, and wrote the manuscript. A.J.S. contributed to the study design, and assisted with experiments. J.T.R. assisted with imaging. P.N.V. and C.R.S. assisted with mouse experiments. R.J.C. contributed to funding and writing of the manuscript. M.J.S. facilitated human specimen collection, and contributed to funding and writing of the manuscript. C.L.S. supervised the research, contributed to the study design and writing of the manuscript. K.S.L. conceived the study, analyzed the data, wrote the manuscript, obtained funding, and supervised the research.

\section{COMPETING INTERESTS}

C.L.S. receives research funding from Bristol Myers Squibb and Janssen and a personal fee from Merck \& Co. in 2019 for an advisory role. The authors declare that there are no other competing interests. 


\section{ADDITIONAL INFORMATION}

Supplementary information is available for this paper at https://doi.org/10.1038/ s41522-020-00143-x.

Correspondence and requests for materials should be addressed to K.S.L.

Reprints and permission information is available at http://www.nature.com/ reprints

Publisher's note Springer Nature remains neutral with regard to jurisdictional claims in published maps and institutional affiliations.
Open Access This article is licensed under a Creative Commons Attribution 4.0 International License, which permits use, sharing, adaptation, distribution and reproduction in any medium or format, as long as you give appropriate credit to the original author(s) and the source, provide a link to the Creative Commons license, and indicate if changes were made. The images or other third party material in this article are included in the article's Creative Commons license, unless indicated otherwise in a credit line to the material. If material is not included in the article's Creative Commons license and your intended use is not permitted by statutory regulation or exceeds the permitted use, you will need to obtain permission directly from the copyright holder. To view a copy of this license, visit http://creativecommons.org/licenses/by/4.0/.

(c) The Author(s) 2020 